\title{
Gestión económica vinculada con la innovación y adquisición de tecnológica en los emprendimientos emergentes de negocio tipo PYME*
}

\author{
Alizo, María Alexandra ** \\ Graterol, Angela *** \\ Chávez, Belkis ****
}

\section{Resumen}

La presente investigación tuvo por objetivo analizar los factores de gestión económica vinculados con la innovación y adquisición tecnológica para el emprendimiento emergente de negocio (EEN) tipo Pequeñas y Medianas Empresas (PYME) del municipio Maracaibo del estado Zulia, durante el período 1999-2006. La metodología utilizada, fue de campo, descriptiva y exploratoria, fundamentada en los objetivos de la investigación y, cualitativa, ya que utilizó algunas técnicas de análisis propias de las representaciones sociales, como lo es la técnica de saturación, la cual consiste en agotar las respuestas de los actores del contexto a indagar. Los resultados denotaron algunas características en los emprendimientos emergentes tipo PYMES bien significativas, entre otras que no se disponen de equipos altamente tecnificados en el desarrollo de los procesos productivos, son muy escasos los que desarrollan innovación tecnológica y establecen alianzas estratégicas con organismos generadores de ciencia, tecnología e innovación, aún cuando existe consciencia plena de la importancia de los factores citados en la gestión económica del emprendedor emergente de este municipio.

Palabras clave: Gestión económica, innovación, adquisición tecnológica, emprendimiento emergente tipo PYME.

Recibido: 20-10-09. Aceptado: 25-03-10

* Se agradece el apoyo financiero aportado por el Consejo de Desarrollo Científico y Humanístico de la Universidad del Zulia (CONDES), al Centro de Enseñanza Mateo López por la logística prestada y al Doctor Azcoul Nasser, profesor de la Universidad de los Llanos por su asesoría.

** MgSc en Gerencia de Empresas. Investigador - Profesor FCES/LUZ. Coordinadora de Planificación de LUZ. Candidata a Doctora en Ingeniería y Administración de las Organizaciones en la Universidad Politécnica de Madrid en convenio con LUZ. E-mail: marialexa4@hotmail.com marialexa110@yahoo.com.

*** MgSc en Finanzas y Macroeconomía. Investigador - Profesor FCES/LUZ. Candidata a Doctora en Ingeniería y Administración de las Organizaciones en la Universidad Politécnica de Madrid en convenio con LUZ. E-mail: agraterol@hotmail.com - agraterol60@yahoo.com.

**** Especialista en Contaduría. Investigador - Profesor FCES/LUZ. Coordinadora del Programa de Post grado en Administración del Sector Salud. Consejera de Escuela. Coordinadora del Despacho Decanal FCES/LUZ. E-mail: chavez.belkis@gmail.com. 


\title{
Economic Management Linked to Technology Innovation and Acquisition in Emerging Business Enterprises of the SME type
}

\begin{abstract}
The objective of this study was to analyze the economic management factors linked to technology innovation and acquisition for the emerging business enterprise (EBE) of the Small and Medium Enterprise (SME) type in the Maracaibo Municipality, State of Zulia, from 1999 to 2006. The methodology used was a descriptive, exploratory field study based on the research objectives; it was also qualitative, since it used some analysis techniques belonging to social representations, such as the saturation technique, which consists of exhausting actor responses in the context to be investigated. Results denoted some very significant characteristics in emergent business enterprises of the SME type; among others, they do not have highly technified equipment for developing productive processes, very few develop technological innovation and establish strategic alliances with organisms that generate science, technology and innovation, even when full awareness exists of the importance of the aforementioned factors in economic management for the emerging entrepreneur in this municipality.
\end{abstract}

Key words: Economic management, technological acquisition, SME-type emerging enterprise.

\section{Introducción}

El ámbito actual, dinámico, flexible y de supeditación tecnológica, genera necesidades de ajustes y adaptaciones de la ciencia a disposición y uso de los requerimientos reales de la sociedad. De esta manera, es de imperiosa necesidad el buscar alternativas que faciliten la dinámica social y, sobre todo, que la practicidad de ésta se constituya en un constructo o vía para catalizar los esfuerzos investigativos en correlación a los requerimientos que demandan las sociedades.

Venezuela desde principios de los años ochenta atravesó una crisis que, en primera instancia era considerada de carácter coyuntural y que, hasta principios del siglo XXI, se le comenzó a denominar crisis larga estructural. Esta situación llevó al país a replantearse una serie de lineamientos y acciones en la búsqueda de soluciones. La necesidad de reactivar, diversificar, recuperar y estabilizar el sistema económico ha conllevado a evaluar la necesidad de compartir responsabilidades de los diferentes actores del sistema, para encontrar nuevos caminos que permitan superar la problemática actual.

En este contexto, los economistas han hablado de la importancia de la inversión privada como agente multiplicador del aparato productivo nacional. Es así como surge el proyecto de estímulo a los Emprendedores Emergentes de Negocios (EEN) como posible herramienta de apoyo para contribuir a gestar nuevas oportunidades de empleo y fomento a las iniciativas emergentes, de manera que permitan construir posibilidades alternas para diversificar y crear competencias distintivas, en otras áreas potenciales, actualmente vírgenes, en las que se dispone de ventajas comparativas.

De este modo, en el año 2002 se genera en Venezuela dentro de los gru- 
Gestión económica vinculada con la innovación y adquisición de tecnológica...

Alizo, María Alexandra; Graterol, Angela y Chávez, Belkis

pos intelectuales el deseo de indagar un poco más sobre cómo lograr el desarrollo y estímulo, de la entonces llamada "economía popular" (hoy conocido con el argot de "desarrollo endógeno" que contempla a las pequeñas y medianas iniciativas emergentes de nuevos empresarios), para generar una masa de inversiones autónomas que contribuyesen a crear un proceso de acumulación de capital nacional o nativo para solventar los problemas de déficit de producción interna en el país y resolver el problema del desempleo.

Es a partir de esas inquietudes generalizadas de los actores sociales de este país (empresarios, políticos, economistas, sociólogos, entre otros), sobre todo aquellos que hacen vida activa y tienen capacidad de intervenir, sea por el papel que desempeñan en el juego político, social, económico, cultural o cualquier otro rol ejercido, que se comienza a concentrar la atención en tratar de fortalecer, mediante un conjunto aplicado de políticas, la masa productiva nacional, de modo de erigir nuevas formas de organización para la creación de nuevas empresas.

De esta manera, surgen Decretos con Fuerza de Ley para el estímulo financiero y el apoyo económico, no solamente a las pequeñas y medianas empresas nacionales tradicionales, sino a otras denominadas cooperativas con razones sociales equivalentes para sus grupos asociados, que buscan expandir los beneficios a una mayor cantidad de personas o empresarios emprendedores.

En función de estas referencias y ante los cambios que acometía el actual gobierno, además de las tendencias mundiales de esgrimir los esfuerzos gu- bernamentales para dar apoyo a los emprendimientos emergentes de los diferentes países, surge y se encamina la inquietud del grupo investigador de este trabajo a indagar un poco más sobre las características que asumen algunos factores de gestión económica en los EEN, dentro del Municipio Maracaibo del Estado Zulia de Venezuela.

Por otro lado, no se debe olvidar que la economía es un área del conocimiento para y al servicio de la sociedad, por lo tanto, la sistematización de ésta puede orientar y contribuir para que los EEN dirijan sus esfuerzos y acciones en forma programada y hacia áreas potencialmente productivas y generadoras de impactos positivos en la zona, con el fin de coadyuvar a una gestión óptima en función de la utilización efectiva de los recursos disponibles. La toma de decisiones acertadas podría ser un recurso a disposición de quien conoce la realidad de su entorno y, poder contar con un conocimiento sobre las fallas actuales que acometen las iniciativas emergentes, respecto a las adquisiciones tecnológicas y la forma como se introduce o no la innovación, podría representar un componente distintivo entre el éxito y no, de un negocio emergente.

De este modo, la presente investigación se concentra en analizar los factores de gestión económica vinculados con la Innovación y Adquisición Tecnológica para el ENN tipo PYME, del Municipio Maracaibo del Estado Zulia, en Venezuela, durante el período 1999-2006.

La investigación se justifica ya que pretende facilitar la toma de decisiones gerenciales del emprendimiento, al enfrentar asertivamente la dinámica coyun- 
tural actual en este sector vinculadas a las variables objeto de estudio, no sólo en el Municipio Maracaibo del Estado Zulia, sino en todo el país.

Asimismo, el mercado venezolano requiere impulsar y desarrollar la producción de bienes y servicios no petroleros con programas y proyectos bien fundados y, a futuro, el crear un sistema de gestión económica estratégica autónoma que permita orientar a las nuevas generaciones de emprendedores, para así poder hacer un mejor uso de los recursos públicos orientados a programas de incentivos a la inversión, tomando decisiones atinadas de acuerdo a la nueva realidad social y a las prácticas gerenciales modernas.

Para el logro de este objetivo se aplicó el instrumento denominado Gestión Económica en los Emprendimientos Emergentes de Negocio (GESECON$E E N)$, el cual contó con un total de 97 ítems, aplicado a una población PYMES compuesta por 52.791 gerentes, ubicados en los tres sectores productivos (primario, secundario y terciario), de la cual se extrajo una tipificación de muestra de 2.112 gerentes que permitió determinar los factores de gestión económica relevantes para los EEN en el Municipio Maracaibo, durante el período de estudio. De este modo, se pudo lograr identificar los componentes de carácter económicos que son importantes en la gestión y toma de decisiones empresariales.

Cabe señalar, que para el diseño del instrumento GESECON-EEN se operacionalizaron las variables por dimensión, subdimensión e indicadores mediante las siguientes técnicas: contacto con expertos, mesas de trabajo y la revi- sión teórica conceptual a través de autores que le dieron sustento a este estudio, tales como: Keynes (1937), Fisher (2006), Stanley (1989), Stoner (1994), Dillard (1980), entre otros, y analizando las cualidades de la experiencia en el campo, en la esencia misma del fenómeno, en este caso, la gestión económica en los EEN del municipio Maracaibo.

Asimismo, para obtener la población total de PYMES en el municipio Maracaibo se utilizaron directorios provenientes de diferentes fuentes, entre ellas: del Instituto Nacional de Estadísticas de la República Bolivariana de Venezuela (INE), la Cámara de Industriales del Zulia, Cámara de Comercio del Zulia, Cámara de Construcción del Zulia, Unión de Contratistas del Zulia, Unión de transportistas de Maracaibo, Ministerio para la Economía Popular, Corporación para el desarrollo de la Región Zuliana (CORPOZULIA) y otros directorios proporcionados por organizaciones de otorgamiento de financiamiento, tales como: el Fondo de Crédito Industrial, Instituto Nacional de Desarrollo de la Pequeña y Mediana Industria en Venezuela (INAPYMI), Sociedad de Garantías Recíprocas para la Pequeña y Mediana Empresa (FONPYME), Banco de la Mujer (BANMUJER) y el Banco Industrial de Venezuela (BIV).

Dado lo heterogéneo de la base de datos, se necesitó hacer un estudio preliminar exhaustivo para eliminar a las organizaciones cuyo inicio de actividades no fuese en el período citado (1999-2006) y anexar aquellas otras empresas que, aún cuando estaban ubicadas en las adyacencias a este municipio, su mercado meta y la comercialización lo constituía la ciudad de Maracaibo del estado Zulia, cu- 
Gestión económica vinculada con la innovación y adquisición de tecnológica...

Alizo, María Alexandra; Graterol, Angela y Chávez, Belkis

yos habitantes supera el número de 2.600.000 personas (es la segunda ciudad de Venezuela y base de la actividad petrolera del país). De este modo, durante esta parte preliminar, se segmentó la muestra sectorialmente por actividad económica, para luego hacer un muestreo aleatorio simple utilizando las tablas de números aleatorios, conforme al porcentaje establecido a cada sector y previa identificación de la actividad productiva del negocio y enumeración del directorio. La fiabilidad de la muestra para que tuviese las mismas características que la población total, fue del 99 por ciento.

Por su parte, para el cumplimiento del presente nivel de objetivos, se dividió el proceso de investigación en dos fases, en las cuales se ordenaron técnicas de estudio descriptivo-exploratorio en base a la observación documental y al trabajo de campo:

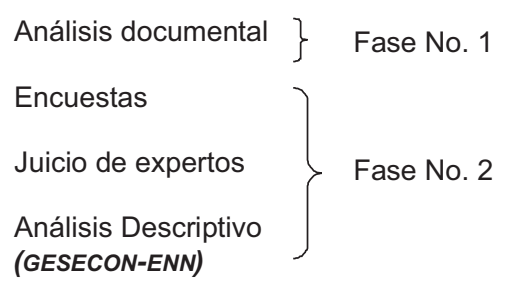

En la fase No. 1, la observación fue la herramienta fundamental para obtener información y sirvió como sustento a la planificación sistemática del registro y control de la revisión conceptual vinculada con el estudio. De acuerdo a lo antes expuesto, la primera tarea fue la de realizar una selección exhaustiva de las teorías de soporte, cuya medición fuera posible, validando los supuestos que involucran dichos conceptos y cuyo producto fue el instrumento GESECON-EEN.
Dicho cuestionario fue revisado en la fase No. 2 por cinco expertos: cuatro en el área de gestión y uno en metodología; con la finalidad de ser luego suministrado a la población en dos momentos, el primero para obtener el grado de confiabilidad del instrumento, para lo cual se llevó a cabo una prueba piloto a 107 personas integrantes del universo de población, pero que no formaran parte de la muestra de PYMES seleccionadas, obteniendo como resultado un Coeficiente de Alfa - Cronbach de 0.91, valor éste que indica que hay consistencia en la confiabilidad del instrumento, ya que se considera que los ítems miden los indicadores y las dimensiones de las variables. El segundo momento fue el de aplicación del cuestionario a los 2.112 gerentes para la obtención de los datos.

Para garantizar la participación intersectorial de todos los EEN, y conocer a ciencia cierta los factores relevantes de gestión económica en cada una de estas actividades productivas, se procedió a extraer en forma igualitaria e intencional los emprendimientos intersectoriales, por lo cual la muestra presentó una distribución porcentual equivalente al treinta y tres punto tres por ciento $(33,3 \%)$ por cada sector de PYMES en el municipio Maracaibo de Venezuela.

Al final de la fase No. 2, se hicieron los análisis descriptivos del instrumento GESECON-ENN, donde se exponen las tendencias porcentuales descriptivas de cada pregunta, según dos escalas: la dicotómica que explora el "sí" o el "no" de los componentes posibles de gestión económica de las PYMES y, la escala múltiple tipo Lícker que aborda la importancia de la sub-dimensión para el emprendedor. 
Asimismo, es importante acotar que en este artículo sólo se analiza parcialmente el modelo de gestión económica, ya que el mismo comprende otras variables, además de la innovación y la adquisición tecnológica, variables éstas a considerar en lo sucesivo.

\section{Gestión en la empresa}

La expresión "gestión" y sus sinónimos (dirección, gobierno y administración) puede definirse como el proceso que logra que las actividades, dentro de un sistema dado, puedan realizarse con eficiencia y eficacia involucrando un equipo determinado de trabajadores, de allí la sintonía que tiene esta expresión con la dirección, ya que denota guiar, encaminar hacia un determinado objetivo (Mauri, 2005: 9).

De este modo, y de forma explícita la gestión se vincula con la eficiencia y la eficacia, ya que uno de los propósitos de la misma es el logro de la efectividad, la cual se puede alcanzar al fusionar los conceptos citados, es decir, mediante la plena y óptima articulación de los recursos disponibles dentro de una empresa y el logro del resultado organizacional.

De forma que en este trabajo se define la gestión empresarial como el proceso de fijación y posterior logro de los objetivos dentro de una unidad o sistema técnico-social abierto, cuya función es la creación de bienes y servicios con el soporte de una toma de decisiones estratégica y efectiva, en cuanto al uso de los recursos y medios organizacionales.

\section{Definición del emprendimiento emergente de negocio}

Hacia la década de los noventa, a las iniciativas emergentes de negocio se les identificaba con las siglas de PYMES o Pequeñas y Medianas Empresas, pero no existía una unidad de criterio con respecto a su denominación, ya que los conceptos que se adoptaban variaban según el tipo de enfoque que se utilizaba.

Es así como para algunos entes institucionales la importancia en el volumen en las ventas, el capital social, el número de personas ocupadas, el valor de la producción o de los activos, eran utilizados como criterios de discriminación entre una u otra forma de categorización. Otros tomaban como referencia el criterio económico-tecnológico, establecían una taxonomía diferenciando entre la pequeña empresa precaria de subsistencia, pequeña empresa productiva más consolidada y orientada hacia el mercado formal o la pequeña unidad productiva con altos niveles de tecnología.

Por su parte, también existían y todavía se presentan otros criterios como el de utilizar la densidad de capital para definir los diferentes tamaños de la micro, pequeña y mediana empresa. La densidad de capital relaciona el valor de los activos fijos, con el número de trabajadores del establecimiento.

En Venezuela, de acuerdo al Instituto de Investigaciones Estadísticas (INE, 2003), una microempresa tiene menos de cinco (5) trabajadores, una pequeña tiene entre cinco (5) y veinte (20) tra- 
Gestión económica vinculada con la innovación y adquisición de tecnológica...

Alizo, María Alexandra; Graterol, Angela y Chávez, Belkis

bajadores, una mediana entre veintiuno (21) y cien (100) trabajadores y una grande tiene más de cien (100) trabajadores.

En la actualidad, el criterio jurídico se basa en dos instrumentos legales: en el Artículo $2^{\circ}$ del Real Decreto con Fuerza de Ley de Creación, Estímulo, Promoción y Desarrollo del Sistema de Micro-Financiamiento, el cual define al Microempresario como aquel que tenga un número no mayor de diez (10) trabajadores y genere ventas anuales hasta de 9.000 unidades tributarias (UT) y, en el Artículo $N^{\circ}$ 3, del mismo Real Decreto con Fuerza de Ley, el cual establece que son Pequeños Industriales aquellos que "llegaren a tener entre once (11) y cincuenta (50) trabajadores al año, así como ventas anuales entre 9.001 a 100.000 unidades tributarias (UT)" (ANRBV, 2002).

Igualmente, el mencionado Real Decreto indica los criterios para distinguir a la Mediana Industria, catalogando a la misma como aquella unidad de explotación económica que tenga anualmente una planta de entre cincuenta y un (51) y cien (100) trabajadores, así como ventas anuales entre 100.001 y 250.000 unidades tributarias (UT).

Sin embargo, ha habido una transformación en el concepto tradicional de las PYMES desde finales del siglo $X X$ y principios del $\mathrm{XXI}$, por una envolvente $\mathrm{y}$ genérica como lo es "Emprendedores de Negocios". Sin embargo, tal como se mostrará no es fácil tratar de precisar el término emprendimiento en el mundo de las organizaciones, ya que son muy versátiles las definiciones e interpretaciones que en los últimos años se le ha dado a esta connotación lingüística, hoy tan en boga.
De tal forma que Hisrish, Peters y Shepherd (2006:7) definen el emprendimiento como una "iniciativa empresarial que tiene por objetivo un proceso de creación de algo nuevo con valor, dedicando tiempo y el esfuerzo necesario, asumiendo los correspondientes riesgos financieros, psicológicos y sociales. Obteniendo las recompensas resultantes de satisfacción e independencia económica y personal".

Por otra parte, Vainrub (2005: 25) conceptualiza el emprendimiento como la combinación de tres factores básicos: "la idea o la oportunidad, los recursos para llevar a cabo la idea, y el empresario que promueve y transforma en realidad la oportunidad. Estos tres elementos se consolidan en un plan, informal o estructurado, dando por resultado una nueva iniciativa empresarial".

Espinoza (1995), expone que el emprendimiento consiste en todas aquellas iniciativas individuales y/o colectivas con capacidad para identificar oportunidades, ubicar y organizar medios y recursos para aplicarlos al desarrollo económico y social sostenible en el ámbito local y regional, el cual implica la disposición de las cualidades del ingenio y de la creatividad por un número significativo del componente humano calificado o no.

El analizar a los autores citados lleva a concluir que existen ciertos componentes comunes en las definiciones del emprendimiento, tales como: crear valor, dedicar tiempo y esfuerzo, asumir riesgos, organizar asertivamente los recursos disponibles con el propósito de lograr un fin práctico y combinar la innovación como un garante del logro y permanecer dentro de un sector productivo determinado por un tiempo. 
De lo anterior se puede decir que para la presente investigación el concepto de Emprendimiento Emergente de $\mathrm{Ne}$ gocio se enmarcará dentro del marco jurídico de las Leyes de la República Bolivariana de Venezuela, que en su Artículo $2^{\circ}$ del Decreto con Fuerza de Ley de Creación, Estímulo, Promoción y Desarrollo del Sistema de Micro-Financiamiento, el cual establece como pequeñas y medianas empresas a todas aquellas organizaciones con un número inferior a 100 trabajadores directos y con ventas anuales menores a 250.000 unidades tributarias (UT) creadas en los últimos diez (10) años (ANRBV, 2002).

Además, se incorpora el que éstos deban reunir a una o un grupo de personas que hayan decidido iniciar una inversión o algún emprendimiento emergente en el mundo de los negocios, lo que incentiva a desarrollar una serie de cualidades intrínsecas para asumir la nueva aventura empresarial, tales como: la innovación, capacidad perceptiva de oportunidades, gerencia proactiva, disposición a asumir riesgos, adopción de sistemas y tecnologías de comunicación vanguardista, visión prospectiva para la gestación de productos y/o bienes y servicios de comercialización exitosa en cualquier lugar del mundo, por parte de iniciativas particulares o colectivas para mantenerse en un mercado cambiante y globalizado.

\section{Gestión Económica en los Emprendimientos Emergentes de Negocio (GESECON-ENN)}

En la era de la Economía del Conocimiento, las empresas toman decisiones empresariales cada vez más desafiantes y complejas, y no es extraño que los ENN del nuevo siglo deban considerar factores diversos como el pensamiento estratégico, la visión prospectiva o futurística sobre el negocio y la realidad que lo rodea, la inteligencia emocional, la constitución de equipos de trabajo proactivos y autodirigidos dentro del proceso de toma de decisiones vinculadas con la organización.

La gestión económica implica adaptarse al entorno económico, cuyos componentes son: mano de obra, tasa de interés, tipo de cambio, disponibilidad de recursos de la zona, adquisición tecnológica, financiamiento público, producción, inversión, innovación y mercado, elementos éstos presentes implícitamente dentro del contexto y proceso de toma de decisiones de cualquier nuevo EEN.

Por lo tanto, la existencia de ambientes económicos adversos o favorables a los nuevos EEN y la forma como los emprendedores manejen o gestionen esos recursos, será un elemento básico para determinar lo efectivo que es el proceso de optimización de los recursos, para lograr los objetivos del negocio dentro de un contexto geográfico, económico-social y con la presencia de un marco jurídico-legal determinado.

De este modo, la gestión económica de esos nuevos emprendedores puede variar de acuerdo al conocimiento e importancia que estos gerentes asuman respecto a estos factores $y$, seguramente, tomen decisiones económicas las cuales incidirán en los resultados a largo plazo de la efectividad del emprendimiento en el país. La presente investigación organiza y sistematiza parcialmente la importancia que los EEN le dan a estos factores. Son estas diferencias lo que permitirá desarrollar el 
Gestión económica vinculada con la innovación y adquisición de tecnológica...

Alizo, María Alexandra; Graterol, Angela y Chávez, Belkis

sistema de gestión económica, cuya versatilidad será determinante y dependerá de estos resultados.

La Figura 1 muestra gráficamente la concepción preliminar del modelo de gestión económica propuesto y se aprecia como éste presenta un doble direccionamiento. Por un lado, se visualiza como los EEN reaccionan ante los factores externos de su entorno económico, en cuanto a los procesos de organizar, planificar, dirigir y controlar las actividades vinculadas con sus unidades productivas, es precisamente esta parte lo que se denomina la gestión de los EEN. Por otro lado, denota como esos factores vinculados a la gestión económica inciden y afectan la toma de decisiones empresariales (sombreado) estableciendo una influencia en el estilo de gestión del emprendedor y, en consecuencia, en la forma que adopte la toma de decisiones de los inversores.
Como el entorno presenta muchas aristas y su generalidad es difícilmente capaz de medirse y establecer estándares de comportamiento, la investigación se centra en la forma cómo los EEN gestionan internamente los factores del entorno económico (para el caso específico de este artículo, los factores son la innovación y las adquisición tecnológica).

\subsection{Gestión Económica Relacionada con la Adquisición Tecnológica}

La tecnología es uno de los factores básicos para el logro del desarrollo endógeno de un sistema económico y, en concreto, para generar una sinergia emprendedora capaz de sustentarse por sí misma, creando los mecanismos pertinentes para lograr la motorización del aparato productivo nacional.

En términos generales, la tecnología se conoce como el proceso a través

Figura 1

Sistema propuesto de gestión económica de los emprendimientos emergentes de negocios.

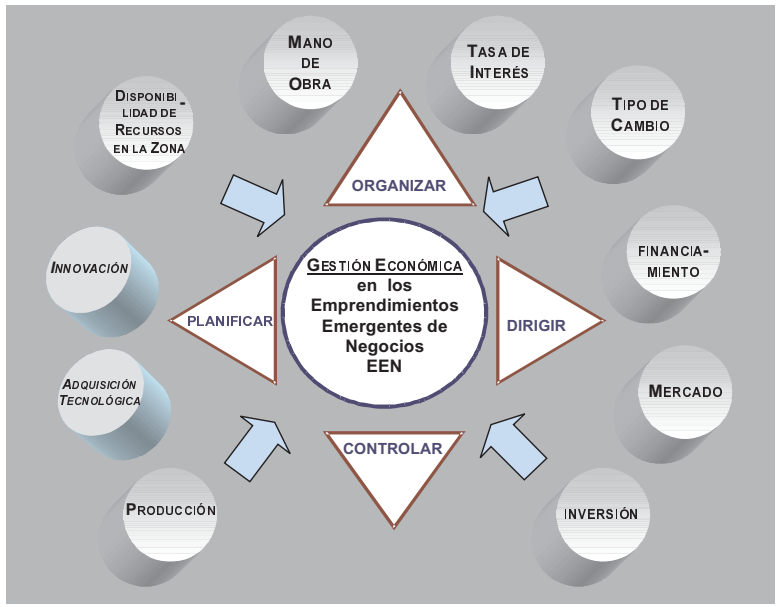

Fuente: Alizo, et al. (2008). 
del cual los seres humanos diseñan herramientas y máquinas para incrementar su control y su comprensión del entorno material. El término proviene de las palabras griegas "tecné", que significa arte u oficio, y "logos", cuya traducción es conocimiento, ciencia o área de estudio; por tanto, la tecnología es el estudio o ciencia de los oficios" (Encarta, 2007).

Según el Diccionario de la Real Academia Española (2006), la tecnología es el conjunto de teorías y de técnicas que permiten el aprovechamiento práctico del conocimiento científico, por lo tanto, se establece que la tecnología es la ciencia aplicada o tecno-ciencia, lo cual sólo será válido para algunas tecnologías, aquellas que están basadas en el saber científico.

Sin embargo, no todas las tecnologías son ciencias aplicadas, ya que existen tecnologías en la agricultura y la ganadería que precedieron a las ciencias biológicas en miles de años y se desarrollaron de un modo empírico (ensayo y error), sin hacer uso del saber científico (Wales et al., 2001).

De modo que la tecnología son los medios para satisfacer las necesidades y deseos humanos, son funcionales, permiten resolver problemas prácticos y en el proceso de hacerlo, transforman al mundo haciéndolo mas previsible, artificial y generando grandes transformaciones sociales y ambientales, cuyos resultados son favorables a unos y a otros no, pero que en definitiva, van afectando el desarrollo natural del planeta tierra.

Mas Herrera (2005:180) es aún mucho más directa cuando establece que la tecnología se encuentra en el "raigambre de la sociedad actual; en un pañal, unos lentes, una medicina, incluso en un arma o cualesquier otro instrumento $u$ objeto que sufra algún adelanto".

Para esta misma autora, la tecnología es la mezcla de tres elementos, que son: la base material, el conocimiento inserto dentro del elemento y los propósitos humanitarios o el sustrato emocional, este último vinculado a los fines últimos de toda nueva creación material. La fusión de la base material y del conocimiento genera los fines y, como la tecnología está íntimamente ligada a las intenciones humanas, ésta se torna infinita y compleja, pro-humana o beligerante, de acuerdo a quienes la promuevan (Mas, 2005).

Por su parte, el análisis Schumpeteriano (1949), que prevaleció hasta más de la mitad del siglo $\mathrm{XX}$, aseguraba que las adquisiciones tecnológicas que se incorporaban a las organizaciones eran las responsables directas de los ciclos económicos o etapas de estancamiento y declive, de modo que refutó la teoría de la ley neoclásica de los crecimientos decrecientes, la cual predecía el agotamiento de las tierras fértiles y el sofocamiento del capital a futuro.

Para Schumpeter (1949) citado por Ondategui (2006) gracias al empresario emprendedor, el Estado y las grandes compañías que internalizan el desarrollo de la ciencia y la tecnología, en su propia organización, al crear, mantener y sustentar las mejoras en los centros organizativos donde se promueve el trío investigación, desarrollo e innovación ( $1+\mathrm{D}+\mathrm{I})$, de forma específica, logrando mejoras en las actividades productivas y en la experiencia histórica empresarial, de forma de contrarrestar efectivamente el efecto nocivo de pérdida de valor de las tierras y los 
Gestión económica vinculada con la innovación y adquisición de tecnológica...

Alizo, María Alexandra; Graterol, Angela y Chávez, Belkis

espacios explotados durante siglos (Ondategui, 2006).

Desde esta perspectiva, serían las empresas, normalmente grandes, quienes toman el liderazgo en la generación tecnológica a nivel mundial mediante la inversión de gigantescas sumas en $I+D+I$, lo cual se traduce a un flujo acelerado y continuo de Innovaciones.

Por consiguiente, el desarrollo económico tendría un carácter dinámico y estaría lleno de discontinuidades al aparecer y aplicar nuevas tecnologías, cuyos objetivos serían: la introducción de un nuevo bien, un nuevo método de producción, la apertura a nuevos y más extensos mercados, las conquistas de nuevas fuentes de energía y aprovisionamiento, y la creación de nuevos métodos organizacionales (Porter, 1990).

De este modo, la adquisición de la tecnología de vanguardia aparece como un proceso de destrucción creativa, a la vez que se comporta como motor del desarrollo económico y regional. Pero, este factor tecnológico no viene determinado de forma únicamente externa como lo planteaban los neoclásicos, si no que es producto de múltiples y complejas decisiones competitivas, tanto de las empresas y sus organizaciones como de componentes externos, como las políticas gubernamentales en materia de promoción y desarrollo científico, formación del recurso humano, apoyo financiero, entre otras; lo que ha permitido democratizar y extender la posibilidades de recuperación tecnológica hacia niveles tradicionalmente excluidos en los diferentes países.

Los emprendedores emergentes de negocio hoy día, podrían incrementar su acceso a tecnologías innovadoras, siempre y cuando aprovechen las oportunidades de las políticas de apoyo a las iniciativas de inversión que promueven los agentes gubernamentales de los países tercermundistas para tratar de equiparar y fortalecer su aparato productivo interno, respecto a los países altamente tecnificados y desarrollados.

Para ello, sería necesario, no sólo tomar decisiones estratégicas respecto al proceso de adquisiciones tecnológicas, sino incluso medir las oportunidades que en materia de fabricación de artefactos o equipos novedosos presenta el mercado en el cual se están introduciendo. De esta manera se establecen un conjunto de etapas para la generación, fabricación y transferencia tecnológica de equipos novedosos para incorporarlos al proceso de producción empresarial, las cuales se precisan a continuación cronológicamente (Wales et al., 2001):

- Identificación del problema práctico a resolver.

- Establecimiento de los requisitos que debe cumplir la solución.

- Principio de funcionamiento, en el cual se estima que eventualmente existen diferentes maneras de resolver un mismo problema.

- Diseño del artefacto, equipo o tecnología vanguardista.

- Construcción de un prototipo.

- Fabricación en serie, en la que se busca la colocación de los productos novedosos en el mercado.

Tal como se ha ilustrado, la adquisición tecnológica es un componente básico e indispensable en toda nueva iniciativa de producción. Esta puede ser un dis- 
positivo competitivo y clave para liderar un sector productivo.

Sin embargo, a pesar de todos los aspectos positivos, entre ellos: el aumento del tiempo de ocio para el hombre, incremento y masificación de la producción, la división y especialización del trabajo, generación de puestos y trabajos novedosos y menos riesgosos, incremento de la productividad, aumento del nivel de vida, reducción de la jornada laboral, éstos también pueden tener en contraposición ciertos efectos negativos. Entre los costos más elevados y nefastos de la época moderna podemos considerar el grave daño ambiental y la falta de responsabilidad social de las organizaciones o empresas.

\subsection{Gestión económica relacionada con la innovación}

La innovación suele concebirse como la acción y efecto de innovar. Desde el punto de vista gramatical, se establece que es, en concreto, el efecto de mudar o alterar algo, introduciendo alguna novedad. Al trasmutar dicho concepto al plano de lo económico - administrativo-organizacional, se puede decir que es la creación o modificación de un producto, proceso productivo, comercialización y/o su introducción futura en el mercado (Encarta, 2007).

Para Mas (2005: 181) "la innovación es cuando se establece algún cambio en los componentes tecnológicos del sistema, es decir, en la base material, el conocimiento o el propósito humano que es lo que conforma la tecnología". Desde esta perspectiva, la innovación posee una frecuencia de cambio inimaginable, puesto que las necesidades humanas son complejas, cambiantes y se construyen en la base de los móviles que promueven la propia innovación científica.

Fischer y sus colaboradores (1989), establecen que la innovación está íntimamente vinculada a los aspectos relacionados al perfeccionamiento de los conocimientos técnicos y, en consecuencia, es el desarrollo de métodos para aplicar los conocimientos existentes en cualesquier área del saber.

Según Kuczmarski (1997) una empresa con mentalidad innovadora representa una actitud novedosa y emprendedora que asumen todos los empleados de una organización, desde el jefe o gerente, hasta los trabajadores por horas. Es un espíritu que penetra y estimula a los individuos al igual que a los equipos, para en forma holística, reafirmar la fe en la creación de novedades, a través de los siguientes componentes: Nuevos mercados, nuevos productos, nuevos segmentos de clientes, nuevos métodos de venta, nuevos negocios, nuevas direcciones estratégicas, nuevos servicios, nuevas formas de liderazgo, nuevas adaptaciones, mejoramiento de productos existentes, entre otras posibilidades que van surgiendo.

El replanteamiento de cada uno de los componentes básicos del marketing moderno es uno de los aspectos elementales que el emprendedor innovador debe establecerse en el momento de penetrar o, simplemente querer comercializar algunos de sus productos.

Igualmente plantea Kuczmarski (1997), que para desarrollar una estrategia de innovación es necesario seguir las siguientes etapas: 
Gestión económica vinculada con la innovación y adquisición de tecnológica...

Alizo, María Alexandra; Graterol, Angela y Chávez, Belkis

- Suministrar un enfoque lógico para crear de manera sistemática algo nuevo.

- Permitir la integración de los puntos de decisión y aprobación dentro del proceso, después de cada etapa.

- Ofrecer un método para dirigir este proceso mediante el seguimiento de los diversos conceptos nuevos que se hallan en desarrollo, en cualquier momento de cada etapa.

Los innovadores son un grupo atrevido, constituyen aproximadamente el $3 \%$ del mercado y son los primeros en incorporar dentro de sus procesos productivos una innovación respecto a las personas que lo adoptan posteriormente.

Según algunos estudios se ha podido evidenciar que los innovadores tienden a ser jóvenes, tienen un nivel social alto y están en mejor posición financiera. Asimismo, éstos tienden a tener relaciones sociales más amplias y más cosmopolitas. Confían más en fuentes impersonales de información, incluyendo las que son externas a su propio sistema social, vendedores $u$ otras fuentes de comunicación personal (Stanton, 1987).

Unas de las características que muchos asocian con los nuevos emprendedores de negocios es la innovación, ya que es ella la que se constituye en el estímulo para iniciar una aventura empresarial. Pero, "no todas son exitosas, ya que por cada caso que una nueva idea creativa lleve al éxito, hay muchas innovaciones que no lo consiguen" (Lambing y Kuehl, 1998: 74).

Cada intento de capitalizar alguna innovación empresarial tiene su propia serie de problemas, es por ello que se puede intuir que no toda innovación lleva al éxito de un negocio, ni garantiza la supervivencia de una empresa. El empresario exitoso necesita de algo más. Es decir, aunque muchas personas sostienen que la innovación es un ingrediente esencial en el espíritu emprendedor (como se aseguró cuando se definió las características de éste), sin embargo, se deben transitar ciertas etapas antes de llevar una idea a la práctica o al mercado.

De este modo, la primera necesidad es que una vez que la idea se ha desarrollado con claridad, debe evaluarse con cuidado en cuanto a los clientes que serán atendidos por el negocio y de la competencia que enfrentarán. El análisis coadyuvado de estos dos componentes permitirá dar respuesta a la interrogante del por qué el negocio debe establecerse o, lo que es lo mismo, justificará la pertinencia de la iniciativa emprendedora.

La segunda necesidad es idear e implementar un plan formal de negocio con decisiones y acciones, independientes, iniciadas estratégicamente e integradas a los procedimientos y debidamente coordinadas.

Cuando falta una estrategia competitiva innovadora, los emprendedores de negocios no cuentan con un marco referencial para entrecruzar las diferentes iniciativas de acción empresarial con un todo coherente, ni con un plan para mantener en armonía el ciclo económico y financiero, con las distintas unidades básicas para desarrollar cabalmente acciones con visión de futuro.

Un plan de negocios es una serie de actividades relacionadas entre sí para el 
comienzo o desarrollo de una iniciativa emprendedora o proyecto innovador con un sistema de planeación tendiente a alcanzar metas determinadas. El plan se desarrolla independientemente el proyecto presente o no innovación. Sin embargo, cuando se le incorpora el componente innovador, el plan de negocio significa un elemento vital para el desarrollo exitoso del proyecto (Pride y Ferrell, 1990).

El plan de negocios debe transmitir a los nuevos inversionistas, a los accionistas y a los grupos financieros, los factores que harán de la empresa un éxito, la forma en la que recuperarán su inversión $y$, en el caso de no lograr las expectativas de los socios, la fórmula parar terminar la sociedad y cerrar la empresa.

El plan de negocios debe justificar cualquier meta sobre el futuro que se fije. Debe ser muy dinámico, por lo que se actualizará y renovará de acuerdo a las necesidades del momento. Asimismo, debe proporcionar un panorama general del mercado y de los requerimientos de la nueva empresa, producto, servicio o, en su caso, de su crecimiento (Kuczmarski, 1997).

Para finalizar el punto, es importante acotar que el emprendimiento emergente no siempre alienta el componente innovador. Existen diferentes estilos de iniciativas emprendedoras, unas con mayor predisposición a asumir nuevos retos vinculados con aplicaciones tecnológicas vanguardistas, otros con adiciones tradicionales en sus procesos productivos. Es menester para esta investigación conocer que tan relevante es el componente innovador en los EEN en la economía venezolana.

\section{Resultados de la investigación}

A continuación se presentan los resultados en cuanto a las variables adquisición tecnológica e Innovación

\subsection{Variable - Adquisición Tecnológica}

Ítems GESECON-ENN: 55-56-5758-59-60-61-62-63-64-65-66-67-68-69

El análisis sobre el estado de la adquisición tecnológica se inicia indagando sobre si están altamente tecnificados los procesos productivos dentro de la organización (ítem 55), para lo cual el cincuenta y nueve punto ocho por ciento (59.8\%) de los emprendedores de tipo PYMES exteriorizó no poseer equipos altamente tecnificados, mientras que el cuarenta punto dos por ciento $(40.2 \%)$ restante informó si contar con alta tecnología (Ver Tabla 3).

En relación a la importancia que le asignaron a este factor los pequeños y medianos productores de las empresas tradicionales emergentes, la gran masa de productores, el cuarenta y siete punto siete por ciento $(47.7 \%)$ ubicó a este componente como de mediana importancia, el diecisiete por ciento $(17 \%)$ de baja y, solamente un acumulado de treinta y cinco punto tres por ciento $(33.2 \%$ y $2.1 \%)$ expresó el considerarlo como un elemento "fuerte" y "muy fuerte" para la toma de decisiones empresariales (Ver Tabla 4, ítem 55).

Esta situación contrasta con lo que generalmente se piensa que es prioridad para los empresarios, quizás la explicación radique en que gran parte de la pequeña y mediana empresa trabaja con ni- 
Gestión económica vinculada con la innovación y adquisición de tecnológica... Alizo, María Alexandra; Graterol, Angela y Chávez, Belkis

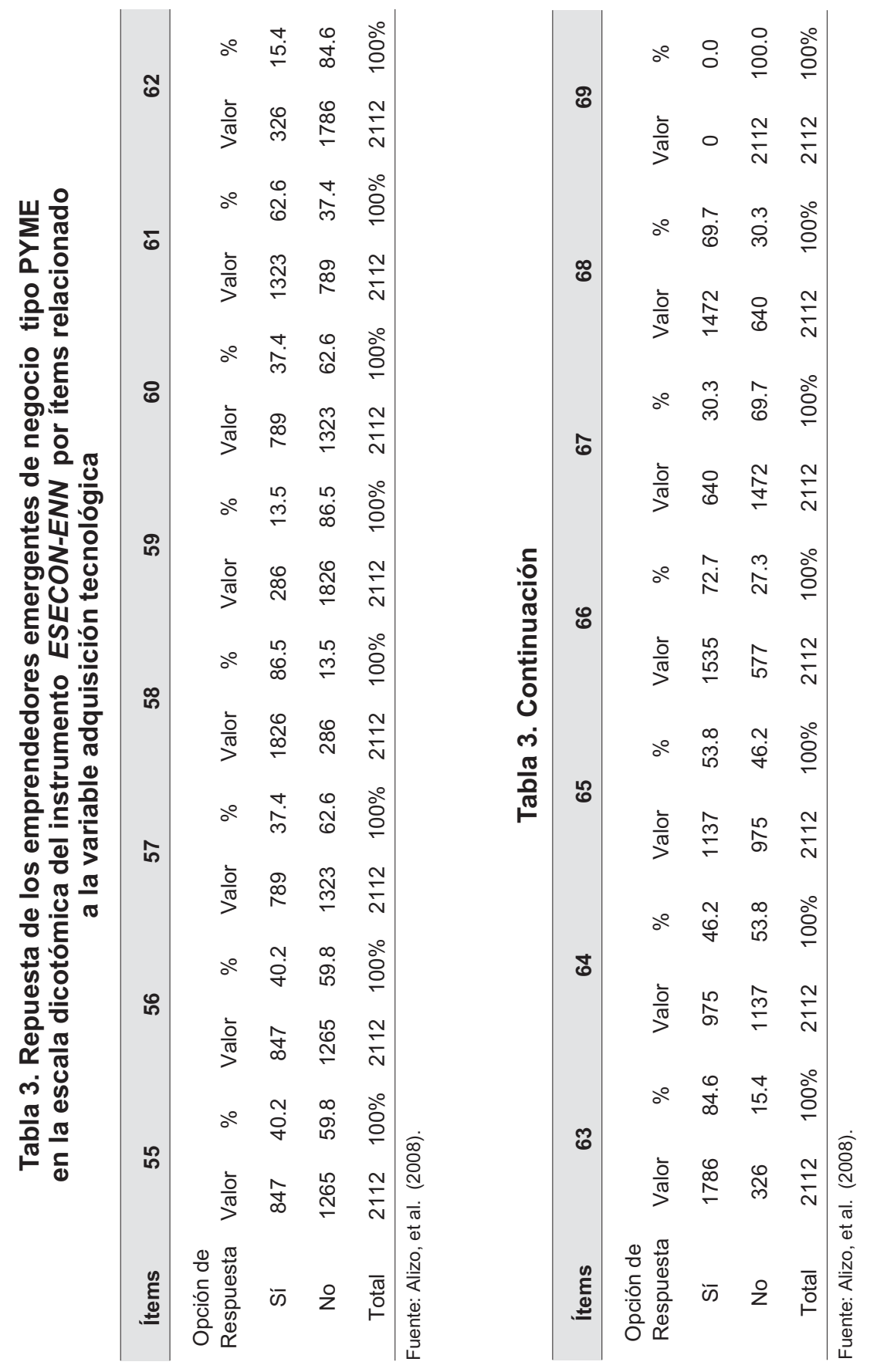



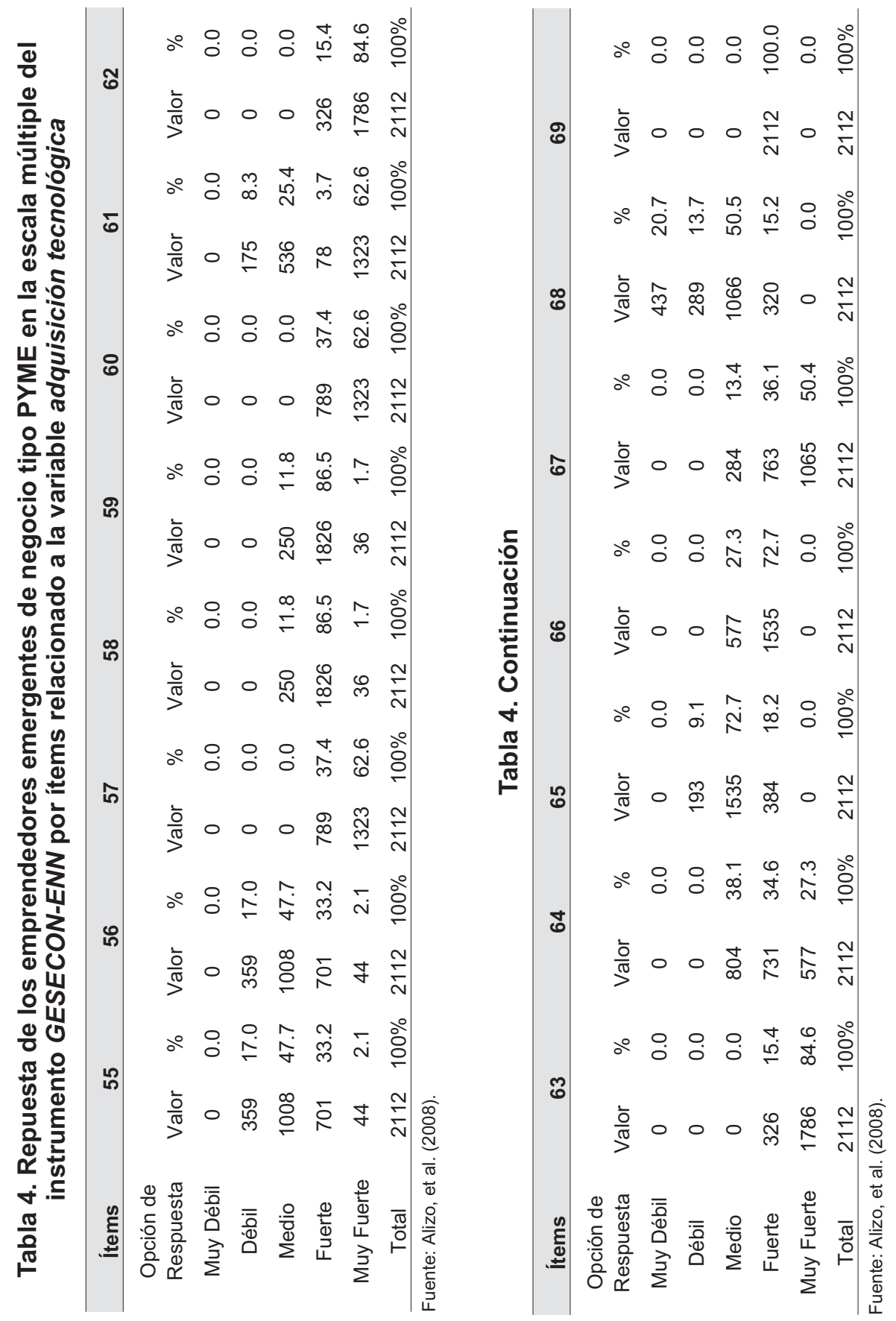
Gestión económica vinculada con la innovación y adquisición de tecnológica...

Alizo, María Alexandra; Graterol, Angela y Chávez, Belkis

veles significativos e intensivos de mano de obra, en consecuencia, la tecnología puede convertirse en un componente secundario.

Al analizar el ítem 56, donde se pregunta si consideran que la empresa utiliza la última tecnología vinculada al área o actividad en la que ella se desempeña, el cincuenta y nueve punto ocho por ciento (59.8\%) de ellos responden que no, y el restante cuarenta punto dos por ciento $(40.2 \%)$ manifiesta si utilizar tecnología vanguardista (Ver Tabla 3). Respecto a la importancia asignada al factor, se denota una clara tendencia acumulativa a considerar a este componente como un factor de mediana relevancia a fuerte, para la masa de emprendimientos de esta naturaleza (Ver Tabla 4).

Asimismo, en el ítem 57 se les pregunta a los empresarios si la capacidad tecnológica instalada es adecuada al tamaño real del mercado, el treinta y siete punto cuatro por ciento (37.4\%) de los encuestados planteó que sí, pero un significativo sesenta y dos punto seis por ciento $(62.6 \%)$ contestó no contar con un volumen cimentado de capacidad tecnológica acorde a su mercado (Ver Tabla 3, ítem 57).

Con respecto a la importancia asignada a este factor los emprendedores están concientes de su relevancia y, esto se hace evidente cuando asignan, acumulativamente, el cien por ciento (100\%) a "fuerte" y "muy fuerte", en la escala múltiple, para el movimiento emprendedor tipo PYMES (Ver Tabla 4, ítem 57).

En este mismo orden de ideas, al abordar el ítem 59, en el cual se les consulta a los inversores de negocios si éstos están utilizando a plenitud toda la capacidad instalada en los procesos de produc- ción de bienes y/o servicios en sus organizaciones, el ochenta y seis punto cinco por ciento (86.5\%) dijo no hacerlo y, solamente un trece punto cinco por ciento $(13.5 \%)$ de ellos expresó que sí. Esto denota escasa productividad dentro de los sectores empresariales tipo PYMES, Io cual a largo plazo podría significar el cierre o pérdida para las estructuras sectoriales de producción de bienes y servicios (Ver Tabla 3).

Por otra parte, al preguntarles sobre la importancia de este factor, el ochenta y seis punto cinco por ciento (86.5\%) de los encuestados opinó que es un componente fuerte para el emprendimiento emergente (Ver ítem 59, Tabla 4).

Acerca del acceso de estos inversores a la alta tecnología que les permitiese desarrollar eficientemente sus procesos productivos, el sesenta y dos punto seis por ciento $(62.6 \%)$ de los emprendimientos planteó que no les fue fácil y, el restante treinta y siete punto cuatro por ciento (37.4\%) expresó que sí. Esto concuerda con la aseveración hecha por un grupo de inversores, el cuarenta por ciento $(40 \%)$, que manifestó disponer de tecnología avanzada y adecuación con su mercado (ítems 56 y 57). Para el cien por ciento acumulado ( $37.4 \%$ y $62.6 \%$, respectivo) de los empresarios, este es un factor "fuerte" y "muy fuerte" para la masa de estímulo del negociador (Ver ítem 60 y 61 , Tablas 3 y 4 ).

Siguiendo con el análisis de las adquisiciones tecnológicas de los emprendimientos tipo PYMES, cuando se les consultó a los inversores emergentes si les resultaba fácil la sustitución tecnológica de la actual tecnología por una nueva y vanguardista, el ochenta y cuatro punto 
cinco por ciento (84.5\%) de los empresarios planteó que no le era fácil, mientras que el quince punto cinco por ciento (15.5\%) expresó que sí y, en ambos casos (ítems 61 y 62 ), plantearon que era un factor "fuerte" $(15,5 \%)$ y "muy fuerte" $(84.5 \%)$ como componente de gestión económica para el desarrollo emprendedor (Ver ítem 62 y 63, Tablas 3 y 4).

Al indagar en este grupo poblacional en PYMES tradicionales si les resultaba fácil, mediano o difícil procurarse de servicios de mantenimiento para la tecnología incorporada dentro de sus organizaciones, los resultaron fueron los siguientes: para el cuarenta y seis punto dos por ciento $(46.2 \%)$ de los encuestados, ha sido fácil buscar servicios de mantenimiento tecnológico, para el cincuenta y tres punto ocho por ciento (53.8\%), por el contrario es difícil la procura de este servicio $y$, el setenta y dos punto seis por ciento $(72.6 \%)$ expresó que les fue medianamente sencillo obtener servicios de mantenimiento tecnológico, según las escalas dicotómicas de los ítems 64, 65 y 66 (Ver Tabla 3).

En relación a la importancia del factor, los resultados fueron convergentes para los tres ítems considerados en el análisis anterior, de modo que los emprendedores manifestaron lo siguiente: para el primer ítem, el 64, una escala mayoritaria de "medio" y "fuerte" en valores acumulativos de setenta y tres por ciento (medio $38.1 \%$, fuerte $34.6 \%$, total acumulado $72,7 \%$ ), para el segundo ítem, el 65, un valor acumulado de noventa y uno por ciento (medio $72,7 \%$, fuerte $18,1 \%$, total acumulado $90,8 \%$ ) en las opciones valorativas de "medio" y "fuerte" y, en el ítem 66 , los resultados mostraron tendencias a establecerse en las calificaciones de "medio" y "fuerte" en un cien por ciento acumulado (medio $27.4 \%$, fuerte $72.6 \%$, total $100 \%$ ). Estas tendencias permiten denotar que los emprendimientos consideran a las facilidades o no de procura de servicios de mantenimiento como un elemento entre mediana y fuertemente importante como factor de gestión económica para el emprendimiento (Ver Tabla 4).

Ahora bien, los tres últimos ítems $(67,68$ y 69$)$ de la adquisición tecnológica también se analizarán en forma conjunta, debido a que ellos se encuentran muy vinculados. En ellos se les preguntaba a los directivos de los emprendimientos tipo PYMES si el origen de los servicios de mantenimiento de la tecnología incorporada en sus procesos productivos era nacional (ítem 67), extranjero (ítem 68) o mixto (ítem 69), para lo cual los emprendedores respondieron lo siguiente: el treinta punto cuatro por ciento $(30.4 \%)$ respondió que los servicios de mantenimiento tecnológicos eran netamente nacionales, el setenta y nueve punto seis por ciento $(69.6 \%)$ que éstos eran extranjero y el cien por ciento (100\%) que no tenían servicios mixtos respecto a la tecnología (Ver Tabla 3).

Al analizar el nivel de importancia que le asignan los emprendimientos tipo PYMES al origen de los servicios de mantenimiento, se pueden visualizar resultados bien interesantes: respecto al ítem 67 donde se establece que el 30 por ciento del origen de los servicios era nacionales, se calificó al factor mayoritariamente entre "fuerte" y "muy fuerte" (fuerte 36,2\%, muy fuerte $50,4 \%$, total acumulado $88,6 \%$, Tabla 4). Por otra parte, en el ítem 68 , en el que se obtenía como resultado 
Gestión económica vinculada con la innovación y adquisición de tecnológica...

Alizo, María Alexandra; Graterol, Angela y Chávez, Belkis

que el 70 por ciento del origen de los servicios de mantenimiento tecnológico eran de carácter extranjero, el nivel de relevancia para el emprendedor de este factor se reducía, concentrándose las respuestas en los niveles de "muy débil" y "medio" en un setenta y uno por ciento ("muy débil" $20,7 \%$, "medio" $50,5 \%$, total acumulado $71,2 \%$, Tabla 4 ).

Finalmente, para el ítem 69, donde se plantea la no existencia en el grupo de entrevistados de emprendimientos que utilicen servicios de origen mixtos de mantenimiento tecnológico, se considera también el factor como un componente "fuerte" para el cien por ciento de los inversores encuestados. Esto denota que los empresarios clasifican como una debilidad el hecho de no disponer de servicios mixtos, sino de tener que depender de los extranjeros en materia de procura de servicios de mantenimiento tecnológico, aún cuando, contradictoriamente, ellos mismos expresaron en un 73 por ciento que les era medianamente fácil obtener este servicio (Ver ítem 66, Tablas 4 y 5).

\subsection{Variable - Innovación:}

Ítems GESECON-ENN: 55-56-6061-63-83-84-85-86-87-88-89-90

Antes de iniciar el análisis de esta variable, se hace pertinente acotar que existen unos ítems que ya fueron analizados en la sección precedente, en consecuencia, en este apéndice sólo se analizarán los que no hayan sido evaluados. No obstante, debe quedar claro que son dimensiones compartidas por estas variables, esto debido a la interconexión e interdependencia que existe en gran parte del sistema de gestión económica, en concre- to entre estas dos variables. A continuación la discusión de los resultados.

Al indagar sobre si las unidades productivas de bienes y/o servicios eran altamente innovadoras respecto a los productos (bienes y/o servicios) que ofrecían en sus mercados, el cuarenta punto ocho por ciento $(40.8 \%)$ de las empresas estudiadas nos informaron que si, pero un cincuenta y nueve punto dos por ciento $(59.2 \%)$ expresó que sus productos no eran novedosos. Asimismo, cuando se evaluó la importancia del factor en la escala múltiple, el mismo fue calificado de "fuerte" (59.2\%) y "muy fuerte" (40.8\%) por los emprendedores encuestados (Ver ítem 83, Tablas 5 y 6).

Cuando se les consultó sobre si sus organizaciones desarrollaban investigación para mejorar los actuales procesos de producción, sólo un once punto tres por ciento $(11.3 \%)$ indicó que si, la gran masa de emprendimiento tipo PYMES expresó que no (88.7\%). Por otra parte, cuando se les pregunta sobre la importancia de este factor, un representativo cuarenta y cuatro punto cuatro por ciento $(44,4 \%)$ le asigna un valor medio, lo cual indica que un porcentaje representativo de empresarios venezolanos no son capaces de captar la suma importancia de este factor para su emprendimiento (Ver ítem 84, Tablas 5 y 6).

El ítem 85, investiga si las empresas poseen algún departamento de investigación o dedican alguna partida para el desarrollo de nuevos productos y tecnologías, los resultados fueron desconcertantes ya que sólo un nueve punto nueve por ciento $(9,9 \%)$ de los emprendimientos disponían del mismo y, un noventa punto uno por ciento $(90.1 \%)$ care- 
Revista Venezolana de Gerencia, Año 15, No. 51, 2010

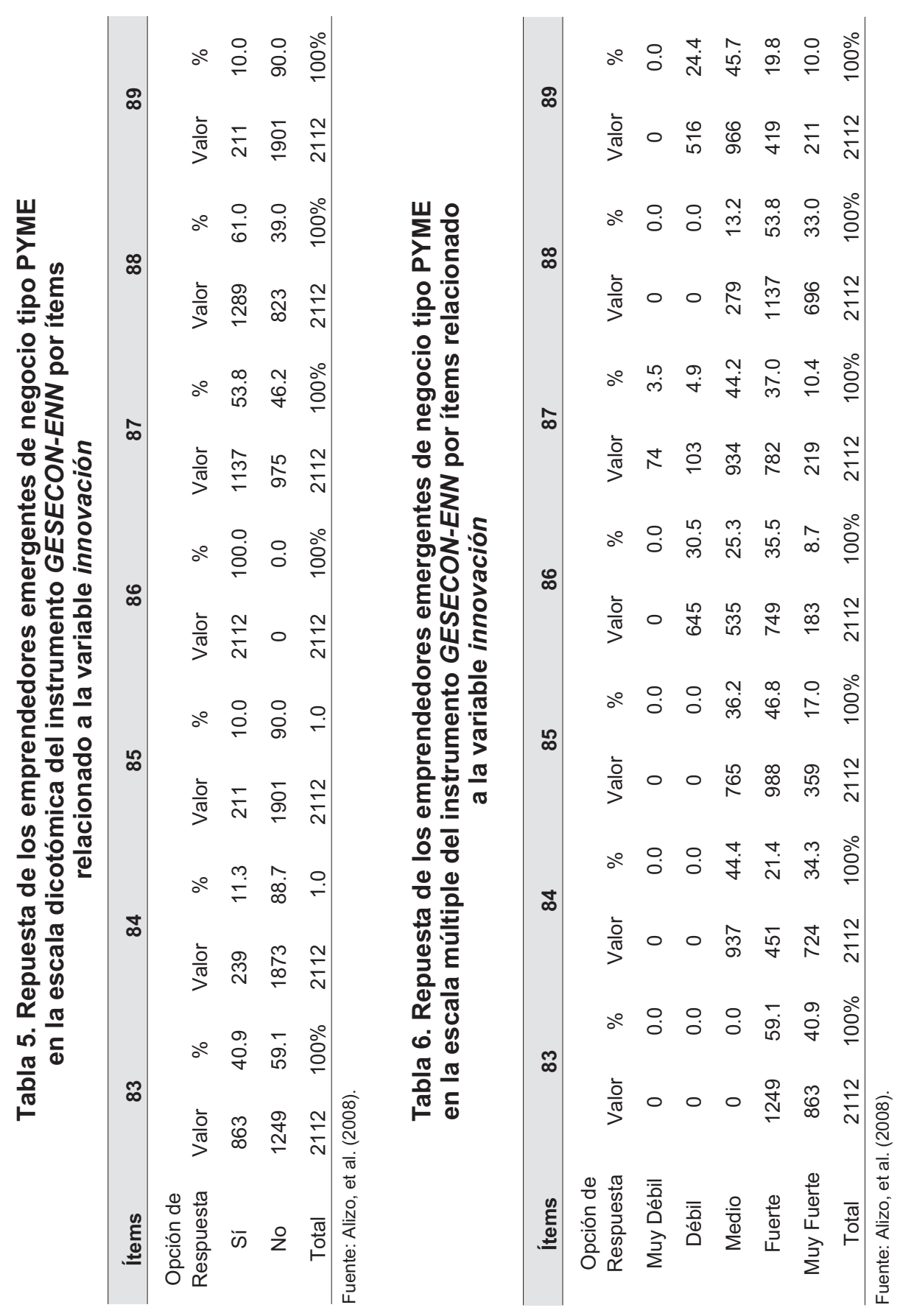


Gestión económica vinculada con la innovación y adquisición de tecnológica...

Alizo, María Alexandra; Graterol, Angela y Chávez, Belkis

El ítem 85, investiga si las empresas poseen algún departamento de investigación o dedican alguna partida para el desarrollo de nuevos productos y tecnologías, los resultados fueron desconcertantes ya que sólo un nueve punto nueve por ciento $(9,9 \%)$ de los emprendimientos disponían del mismo y, un noventa punto uno por ciento $(90.1 \%)$ carecen del mismo. Asimismo, los encuestaron manifestaron que el factor era "medio" y "fuerte" en un porcentaje acumulativo del ochenta y tres punto uno por ciento $(83,1 \%)$ para el desarrollo emprendedor (Ver ítem 85, Tablas 5 y 6).

Con respecto al ítem 86 , donde se examinaba si las organizaciones contaban con equipos de computación para desarrollar sus procesos productivos, el cien por ciento (100\%) respondió que si y, con respecto a la importancia del factor, para un contingente de encuestados del cincuenta y seis por ciento acumulado (débil 30,5\% y medio $25,4 \%$, acumulado $55,9 \%$ ) lo considera un valor "débil" y "mediano" y, el restante, "fuerte y "muy fuerte" (Ver ítem 86, Tablas 5 y 6).

En este mismo orden de ideas, en relación a si poseían sistemas en redes para comunicación inter-departamental y externa (internet), para facilitar los procesos de comunicaciones con proveedores, clientes, entre otros; las cifras denotaron que aproximadamente un poco más de la mitad de los encuestados si lo poseen, pero alrededor de un cuarenta y seis punto dos por ciento $(46,2 \%)$ no disponen de estos sistemas. El mayor peso calificó a este factor como de importancia "media" para el crecimiento y consolidación del emprendimiento (Ver ítem 87, Tablas 5 y 6).
En relación al ítem 89, en el cual se estudia sobre si las organizaciones tenían algún tipo de contacto con las instituciones generadoras de conocimiento regional para mejorar los procesos productivos actuales (por ejemplo: Universidades, Ministerio de Ciencia y tecnología - FUNDAClTE/ZULIA, INCE, Parque Tecnológico del Zulia, entre otros), el sesenta y uno punto uno por ciento $(61.1 \%)$ de la población analizada respondió que si, mientras que un treinta y ocho punto nueve por ciento (38.9\%) dijo que no. Para el mayor porcentaje de los emprendimientos, el factor es mayoritariamente "fuerte" y "muy fuerte", respecto a su relevancia en este sector, ya que se asume que estos capacitan a sus empleados y personal en esas instituciones (Ver ítem 88, Tabla 5 y 6).

Para finalizar con esta variable, el ítem 89 donde se consulta si la organización realiza aportes al Observatorio $\mathrm{Na}$ cional de Ciencia, Tecnología e Innovación (ONTI) o alguna otra institución pública de esta naturaleza, solo el nueve punto nueve por ciento $(9,9 \%)$ respondió que si y, la mayoría señala a este factor como "débil" y "medio" para el emprendimiento emergente de negocio de esta naturaleza (Ver ítem 89, Tablas 5 y 6).

\section{Conclusiones y Recomendaciones}

Del análisis de las respuestas proporcionadas por los emprendedores emergentes de negocios de naturaleza PYMES, en el aspecto relacionado con las $A D Q U I-$ SICIONES TECNOLÓGICAS, se pudo concluir que los emprendimientos del municipio Maracaibo no poseen equipos alta- 
mente tecnificados para desarrollar los procesos productivos dentro de la organización en las empresas. Asimismo, se manifiesta que es éste un factor débil y de mediana importancia para el inversor.

Por otro lado, un número significativo de emprendedores tipo PYMES plantearon contar con tecnología vanguardista en el área en la que se desempeñan. No obstante desestiman la importancia del factor, lo que se evidencia al clasificarlo como un factor "mediano" para su desarrollo productivo.

Para la mayoría de los negociantes, no existe una adecuación entre la capacidad tecnológica instalada y el tamaño real de sus mercados. Sin embargo, todos coincidieron en calificar a este factor como un componente "fuerte" y "muy fuerte" para el emprendimiento emergente.

Asimismo, la mayor parte de los emprendedores tipo PYMES de este municipio, expresaron trabajar por debajo de la capacidad instalada de sus equipos, lo cual significa niveles productivos bajos que podrían afectar la rentabilidad futura y supervivencia del emprendimiento. Este mismo porcentaje evaluó a este componente como "fuerte" para el desarrollo emprendedor.

Por otro lado, para un porcentaje significativo de los inversores le ha sido fácil el acceso a la alta tecnología, esto es coherente con la aseveración hecha por un grupo de emprendedores PYMES, en concreto el 40 por ciento de ellos, quienes manifestaron disponer de tecnología avanzada y adecuada a su mercado.

Con respecto a la capacidad de sustitución tecnológica de la actual tecnología por una nueva y vanguardista, la gran mayoría indicó ser un proceso difícil en el municipio y todos coincidieron en expresar que es éste un factor "fuerte y "muy fuerte" de gestión económica.

En relación a los servicios de mantenimiento tecnológico, el peso mayoritario de encuestados expresó que les ha sido medianamente sencillo el obtener estos servicios, sin embargo, los inversores consideraron a las facilidades o no de procura de los mismos como un elemento entre "mediana" y "fuertemente" importante como factor de gestión económica para el emprendimiento.

Asimismo, los resultados mostraron que los empresarios califican como una debilidad el hecho de no disponer de servicios de mantenimiento tecnológico mixtos, sino de tener que depender de los extranjeros en materia de procura de servicios tecnológicos, aún cuando ellos mismos expresaron, en un 73 por ciento, que les era medianamente fácil obtener este servicio. Lo anterior hace que el factor de no contar con servicios mixtos para el mantenimiento tecnológico se constituya en un componente "fuerte" en forma unánime para todos los encuestados.

A través del análisis al factor INNOVACIÓN sobre las respuestas proporcionadas por los emprendimientos emergentes de negocio tipo PYMES, se constató que la mayoría de las empresas estudiadas no ofertan productos novedosos para el mercado. No obstante, todas apuntalaron a calificar a este factor como "fuerte" y "muy fuerte".

Por su parte, la mayoría de las empresas emergentes no desarrollan investigación para mejorar los actuales procesos de producción. De modo que un porcenta- 
Gestión económica vinculada con la innovación y adquisición de tecnológica...

Alizo, María Alexandra; Graterol, Angela y Chávez, Belkis

je muy representativo de empresarios no es capaz de captar la importancia de la investigación como factor estratégico para el desarrollo emprendedor tipo PYME.

De esta forma se evidencia que sólo un $10 \%$ de las empresas disponen de departamento de investigación o dedican alguna partida para el desarrollo de nuevos productos y tecnologías. Asimismo, los encuestados subestiman la importancia del factor al calificarlo de valor "medio" y "fuerte" en un porcentaje acumulativo.

Por otro lado, todas las organizaciones poseen equipos de computación para realizar los procesos administrativos; sin embargo, también se subestima el factor ya que la mitad lo consideró entre "débil" y "medio" en cuanto a su importancia y, la otra parte mayoritariamente como un factor "fuerte".

Las cifras denotaron que aproximadamente un poco más de la mitad de los encuestados poseen sistemas en redes para comunicación inter-departamental y externa (internet), para facilitar los procesos de comunicaciones con proveedores, clientes, entre otros.

No obstante, alrededor de un $46,2 \%$ no disponen de estos sistemas. El mayor peso de la población estudiada calificó a este factor como de importancia "media" para el crecimiento y consolidación del emprendimiento, lo cual nuevamente minimiza la importancia de las innovaciones comunicacionales dentro de las estructuras organizativas de estos tipos de emprendimientos.

Resulta interesante ver que la mayor parte de los emprendimientos tienen algún tipo de contacto con las instituciones generadoras de conocimiento regional para mejorar los procesos producti- vos actuales y; para ese mayor porcentaje de emprendimientos, el factor es mayoritariamente "fuerte" y "muy fuerte" respecto a su relevancia, ya que se plantea que éstos capacitan a su personal en estas instituciones, tal como se expresó cuando se indagaba en el "factor trabajo" (componente éste del modelo, pero que no se analiza en el presente artículo, sino en la investigación global).

De esta manera, solamente el 10 por ciento de los emprendimientos realizan aportes económicos o de otra índole al Observatorio Nacional de Ciencia, Tecnología e Innovación (ONTI) o alguna otra institución pública de esta naturaleza $y$, en consecuencia, la gran mayoría señala a este factor como "débil" y "medio" para el emprendimiento emergente de negocio de esta naturaleza.

Finalmente, se recomienda aplicar el modelo de la escuela francesa de análisis estratégico prospectivo (método MICMAC) con la data desagregada por sectores productivos y naturaleza de la organización y, conjuntamente con los criterios de los expertos, procurar los factores "clave" de gestión económica del emprendimiento. Cabe señalar, que la investigación como se presenta en este primer avance, solo pone en evidencia ciertas tendencias muy importantes para alimentar las corridas del método MIC- MAC, pero de modo alguno, presenta resultados definitivos.

\section{Referencias Bibliográficas}

Asamblea Nacional de la República Bolivariana de Venezuela - ANRBV (2002). Ley para la promoción y Desarrollo de la Pequeña y Mediana Industria. Martes 3 de Diciembre de 2002. Gaceta Oficial $N^{\circ} 37.583$. 
Asamblea Nacional de la República Bolivariana de Venezuela - ANRBV (2001). Ley de Creación, Estímulo, Promoción y Desarrollo del Sistema Microfinanciero. Marzo de 2001. Gaceta Oficial $N^{\circ} 37.164$.

Asociación de Academias de Real Academia Española (2007). "Diccionario de la Real Academia Española". Disponible en: http://www.rae.es/. Consulta: 14/ 07/2007.

Constitución de la República Bolivariana de Venezuela (1999). "Portal de la Asamblea Nacional”. Disponible en Internet en: http://www.tsj.gov.ve/legislacion/ constitucion 1999.htm (Consulta: marzo de 2006).

Espinoza, Lamo (1995). Política Social Dirigida a Jóvenes. Ponencia Evento: Gestión Pública Empresarial. UCM Madrid.

Fischer, Stanley; Dornbusch, Rudiger y Schmalensee, Richard (1989). Economía. Editorial McGrawHill/Interamericana de España, S.A. Segunda Edición. Madrid - España.

Hisrich, Robert D.; Peters, Michael P.; Shepherrd, Dean A. (2005). Emprendedores. Editorial Mc Graw Hill. Sexta Edición. Madrid - España.

Kuczmarski (1997). Innovación. Editorial McGraw Hill. Primera Edición. México.

Lambing, Peggy; Kuelhl, Charles (1998). Empresarios Pequeños y Medianos. Editorial Prentice Hall Hispanoameriacana, S. A. México.

Mas H., María Josefina (2005). Desarrollo Endógeno-Cooperación y Competencia. Editorial PANAPO de Venezuela, C.A. Caracas. Primera Edición. Octubre.

Mauri Castelló, Jordi J. (2005). Introducción a la Organización y Gestión de Em- presas. Editorial de la Universidad Politécnica de Valencia. España. Disponible en: http//site.ebrary.com/lid/ bibliotecasebiluzsp/Biblio?\%3Fnosr= $1 \& \mathrm{id}=10074195$ layout=document. Consulta: 04/12/07.

Microsoft ${ }^{\circledR}$, “Enciclopedia Encarta ${ }^{\circledR}$. @ ( ) (19932007)". Disponible por: Software de Microsoft Corporation. Reservados todos los derechos. Año 2007.

Ondategui Rubio, Julio César (2006). "Tecnología Industria e Innovación: Los Parques Tecnológicos de España". Universidad Complutense de Madrid, Disponible en: http://site.ebrary.com/ lib/bibliotecaserbiluzsp/Doc?id10115 420\&ppg=21. Consulta: 14/2/2007.

Porter, Michael (1990). La ventaja Competitiva de Naciones. Editorial Mcmillan. Londres.

Pride, W y Ferrell, O. (1990). Marketing. Decisiones y Conceptos Básicos. Editorial Interamericana. México.

Stanton, William (1987). Fundamentos de Mercadotecnia. Editorial McGraw Hill. Séptima edición. México.

Instituto Nacional de Estadísticas - INE (2007). Disponible en: http://www.ine. gov.ve/ine/venezuelaenmapas/mapasmunicipios.asp? Consulta: 09/12/ 2007.

Schumpeter, Joseph (1949). Teoría del Desenvolvimiento Económico. Fondo de Cultura Económica. México.

Vainrub, Roberto (2005). Convertir Sueños en Realidades - Una guía para emprendedores. Ediciones IESA. Tercera edición. Caracas.

Wales, Jimmy y Sanger, Larry (2001). "Enciclopedia Wikipedia en Red". USA/Versión en Español. Disponible en: http://es.wikipedia.org/wiki/Portada. Consulta: 01/12/07. 